\title{
Notes on biology of alfalfa weevil, Sitona humeralis Stephens, 1831 (Coleoptera: Curculionidae) in alfalfa fields of Iğdır/Turkey province
}

\author{
Celalettin GÖZÜAÇIK ${ }^{* 1}$, Neslihan GÜLTEKINN${ }^{1}$, Antonio J. VELÁZQUEZ de CASTRO²
}

ORCID: 0000-0002-6543-7663; 0000-0002-0139-7391; 0000-0002-5357-4126

${ }^{1}$ Iğdır Üniversitesi, Ziraat Fakültesi, Bitki Koruma Bölümü, 76100, Iğdır, Türkiye

${ }^{2}$ Museo de la Universitat de Valencia de Historia Natural, Calle Doctor Moliner, 50, E-46100 Burjassot, Spain

\begin{abstract}
The adults of Sitona humeralis Stephens, 1831 (Coleoptera: Curculionidae) cause serious damages by feeding on the leaf and shoot parts of the clover plant (Medicago sativa L.), and the larvae on the capillary root and nodocytes. This article was carried out in the clover fields and laboratory in Iğdır province in 2019 - 2020 in order to determine the morphology and biology of $S$. humeralis. In the studies, in order to determine the egg stages of $S$. humeralis, a $1-4 \mathrm{~cm}$ deep topsoil containing clover plants in 5 different parts of the field, a soil plant with a depth of $20 \mathrm{~cm}$ and a surface area of $20 \times 20 \mathrm{~cm}$ to determine the larva, pupa and adult stages were taken with the root with a shovel and examined in the laboratory. As a result of the studies, it was found that $S$. humeralis overwinters in egg, larva and adult stages. Female insects lay some of their eggs in October and November in the autumn, pause their egg laying with the decrease of the air temperature, and continue to lay eggs until the end of March and early May as the weather gets warmer. The eggs were laid on the soil surface in the root zone of the clover plant. While the larvae emerging from the eggs laid in early October are active in the soil until the beginning of November, they spend the winter without feeding until the end of March to the beginning of April as the weather gets colder. The larvae that come out of the eggs laid in the late April - early May and enter the pupa stage in the late May - June. Natural deaths start in April - May in adults who spend the winter, a new generation of adults start to emerge from June and the emergence continues until mid - July. From August to mid - October, the new generation adults spend their rest in the soil around the plant roots. In the studies, it was understood that $S$. humeralis produce a generation a year.
\end{abstract}

Keywords: Sitona humeralis, biological notes, alfalfa, Iğdır

\section{Iğdır ili yonca alanlarında yonca zararlısı, Sitona humeralis Stephens, 1831 (Coleoptera: Curculionidae)'in biyoloji üzerine notlar}

\section{Özet}

Sitona humeralis Stephens, 1831 (Coleoptera: Curculionidae)'in erginleri yonca bitkisinin (Medicago sativa L.) yaprak ve sürgün kısımlarında, larvaları ise kılcal kök ve nodozitelerde beslenerek ciddi zararlar oluşturmaktadır. Bu makalede, $S$. humeralis'in morfolojisi ve biyolojisini belirlemek amaciyla 2019 - 2020 yıllarında Iğdır ili yonca tarlaları ve laboratuvarda yürütülmüştür. Çalışmalarda, S. humeralis'in yumurta dönemlerini belirlemek amacıyla, tarlanın 5 farklı yerinde içinde yonca bitkilerinin de bulunduğu $1-4 \mathrm{~cm}$ derinliğindeki yüzey toprağ 1 , larva, pupa ve ergin dönemlerinin belirlenmesi için $20 \mathrm{~cm}$ derinliğinde ve 20 x $20 \mathrm{~cm}$ yüzey alanına sahip toprak bitki köküyle birlikte kürek yardımıyla alınmış ve laboratuvarda incelenmiştir. Çalışmalar sonucunda, S. humeralis kışı yumurta, larva ve ergin dönemlerinde geçirdiği belirlenmiştir. Dişi böcekler yumurtalarının bir kısmını sonbaharda ekim-kasım aylarında bırakmakta, hava sıcaklığının düşmesiyle birlikte yumurtlamaya ara vermekte havaların 1sınmasıyla birlikte mart sonu ve mayıs ayı başlarına kadar yumurta bırakmaya devam etmektedir. Yumurtalar yonca bitkisinin kök bölgesine toprak yüzeyine bırakılmaktadır. Ekim ayı başlarında bırakılan yumurtalardan çıkan larvalar kasım ayı başlarına kadar toprak

\footnotetext{
* Corresponding author / Haberleşmeden sorumlu yazar: Tel.: +905334728425; Fax.: +904762230049; E-mail: celalettin.gozuacik@igdir.edu.tr

(C) Copyright 2021 by Biological Diversity and Conservation Geliş tarihi: 09.02.2021; Yayın tarihi: 15.04.2021 BioDiCon. 958-0221
} 
içerisinde aktifken, havaların soğumasıyla birlikte mart sonu nisan ayı başına kadar beslenmeden kışı geçirmektedir. Nisan sonu - mayıs başlarında, ilkbaharda bırakılan yumurtalardan çıkan larvalar ise mayıs sonu-haziran ayında pupa dönemine girmektedir. Kışı geçiren erginlerde nisan-mayıs aylarında doğal ölümler başlamakta, haziran ayından itibaren yeni nesil erginler çıkmaya başlamakta ve çıkış temmuz ortalarına kadar devam etmektedir. Ağustos’tan ekim ortalarına kadar yeni nesil erginler bitki kökleri civarında toprak içerisinde dinlenerek geçirmektedir. Çalışmalarda $S$. humeralis' in yılda bir nesil verdiği anlaşılmıştır.

Anahtar kelimeler: Sitona humeralis, biyolojik notlar, yonca, Iğdır

\section{Giriş}

Yonca (Medicago sativa L.), baklagiller (Fabaceae) ailesinde yer alan, derin ve güçlü kök sistemine sahip, çok yıllık önemli bir yem bitkisidir [1]. Yonca sahip olduğu derin kök sistemi sayesinde toprağın havalanmasını ve gevşemesini sağlamakta, erozyonun kontrol edilmesine önemli katkıda bulunarak doğayı korumaktadır. Bu bitki yem bitkileri içerisinde en yüksek besin değerine sahiptir. Besicilikte et ve süt verimini \%30'lara kadar arttırabilmekte ve ayrıca silo ve pelet yemi olarak da kullanılabilmektedir [2]. Yonca, Türkiye'de 2019 y1lı 641213 ha ekim alanıyla oldukça geniş alanlarda yetiştirilmektedir. Iğdır ilinde ise, tarla bitkileri ekilişleri içerisinde toplam 19220 ha ekim alanı ile yaklaşık olarak \%32'lik paya sahiptir [3]. Bakımının ve elde edilen kuru otunun maddi getirisinin yüksek oluşundan dolayı ekim alanları yıldan yıla artış göstermektedir. Yonca bitkisinin verim ve kalitesini olumsuz etkileyen zararlı böceklerden Sitona Germar (Coleoptera, Curculionidae) erginleri yonca bitkisinin yaprak ve tomurcuklarında, larvaları ise kök ve kök nodüllerinde beslenerek ciddi zararlar oluşturmaktadır $[4 ; 5 ; 6 ; 7 ; 8 ; 9 ; 10 ; 11 ; 12]$. Sitona türleri, Akdeniz ülkeleri ve Avustralya'da yonca tarımı yapılan alanlarda ana zararlı durumundadır [8; 13; 14; 15]. Aeschlimann [13], Güney Avrupa'da 20 Sitona türü bulunduğunu bu türler içerisinde $S$. humeralis ve $S$. lineatus'un baskın olduğunu, Dimitrov ve ark. [16; 17] ise, Bulgaristan yonca alanlarında S. humeralis ve S. longulus'u en önemli zararlılar olduğunu bildirmişlerdir. Sitona humeralis ülkemiz yonca alanlarında yapılan sürvey çalışmalarında bulunmuş $[18 ; 19 ; 20 ; 21$; 22] ve morfolojik tanımı yapılmıştır [23]. Ancak yonca alanlarında biyolojisiyle ilgili çalışmalara rastlanılmamıştır.

$\mathrm{Bu}$ çalışma, Iğdır ili yonca alanlarında S. humeralis'in biyolojisini belirlemek amacıyla 2019 ve 2020 yıllarında yürütülmüştür.

\section{Materyal ve yöntem}

Çalışmalar, Iğdır ili yonca plantasyonlarında Sitona humeralis'in yumurta, larva, pupa ve ergin dönemlerini takip etmek için 17.11.2019 (Taşburun), 03.01.2020 (Çalpala, Halıkışla ve Y. Çarıkçı), 04.03.2020 (Taşburun), 27.03.2020 (Yenimahalle), 02.04.2020 (Çalpala), 01.05.2019 (Hakmehmet), 18.07.2020 (Aşağ1 çiftlik), 21.08.2020 (Çalpala) ve 04.09.2020 (Taşburun) tarihlerinde yonca tarlalarında ve laboratuvarda yürütülmüştür. Sitona humeralis ${ }^{\circ}$ in yonca tarlasında yumurtalarının bulunduğu dönemleri belirlemek amacıyla, tarlanın 5 farklı yerinde içinde yonca bitkilerinin de bulunduğu $1 \mathrm{~m}^{2}$ alan $1-4 \mathrm{~cm}$ derinliğinde (yüzey) toprak kazılarak 3-5 litrelik cam kavanoz içerisine alınarak laboratuvara getirilmiştir. Laboratuvara getirilen kavanozların üzerine su ilave edilmiş, 10 - 12 saat bekletilmiştir. Daha sonra her bir numune 2 veya 3 parçaya ayrılarak, bunlar sırasıyla bir huni üzerinde 0.9 mm'lik bir plastik elek içerisine alınarak huni yardımıyla bir beher içerisine yıkanmıştır. Yıkanan sıvı örnekler filtre kâğıdı/tülde süzülmüş ve üstte kalan kısım içerisinde \%10'lık NaCl bulunan 200 ml'lik tüplere alınmış, 2 saat sonra, çözeltinin yüzeyindeki kısım $15 \mathrm{~cm}$ çapında 2 kat filtre kağıdına alınarak damıtılmış su ile birkaç kez yıkanarak NaCl'den arındırılmıştır. Filtre kâğıdı üzerinde kalan kısım stereomikroskop altında incelenerek S. humeralis yumurtaları tespit edilmiştir [24]. Bununla birlikte mart ayında toplanan erginler $\left(5 \circ, 5{ }^{\Uparrow}\right)$ yonca bitkisi ile birlikte laboratuvarda kültüre alınarak yumurtaları elde edilmiştir. Larva, pupa ve ergin dönemlerinin belirlenmesi için tarlanın 5 farklı yerinde yonca bitkisi merkez alınarak 20 x $20 \mathrm{~cm}$ yüzey alanı ve $20 \mathrm{~cm}$ derinliğinde bir toprak bloğu kürek yardımıyla alınmıştır [25]. Alınan toprak örnekleri yerinde incelendikten sonra $5 \mathrm{~kg}$ 'llk şeffaf poşetler içerisinde laboratuvara getirilmiş, $1 \mathrm{~m}^{2}$ 'lik bez üzerine dağıtılarak larva ve pupa dönemleri incelenmiştir. İnceleme tamamlandıktan sonra larva ve pupaların ergin dönemlerini elde etmek amacıyla 5 litrelik cam kavanozlarda kültüre alınmıştır. Toplanan $S$. humeralis erginleri ılık suda 24 saat bekletilerek yumuşatılmış ve yapıştırma kartları üzerine yapıştırılmış, aynı zamanda genital kısımları tanımlamak için diseksiyon yapılarak Leica marka mikroskoba takılan Canon 70D DSLR dijital fotoğraf makinesi kullanılarak fotoğraflanmıştır. Türün teşhisinde orijinal deskripsiyonlar, monograflar, anahtar ve yayınlanmış revizyonlar kullanılmıştır [26; 27]. Türün tanımı stereomikroskop, ölçümleri mikroskoba takılı oküler mikrometre yardımı ile yapılmıştır.

\section{Bulgular}

Sitona humeralis Stephens, 1831, Coleoptera takımının Curculionidae familyasının Entiminae Schoenherr, 1823 altfamilyasının Sitonini Gistel, 1848 tribüsü içerisinde yer alan Sitona Germar, 1817 cinsine ait bir türdür. Çalışmalarda elde edilen sonuçlarla birlikte, türün yayılışı ve konukçu bitkileri aşağıda verilmiştir. 


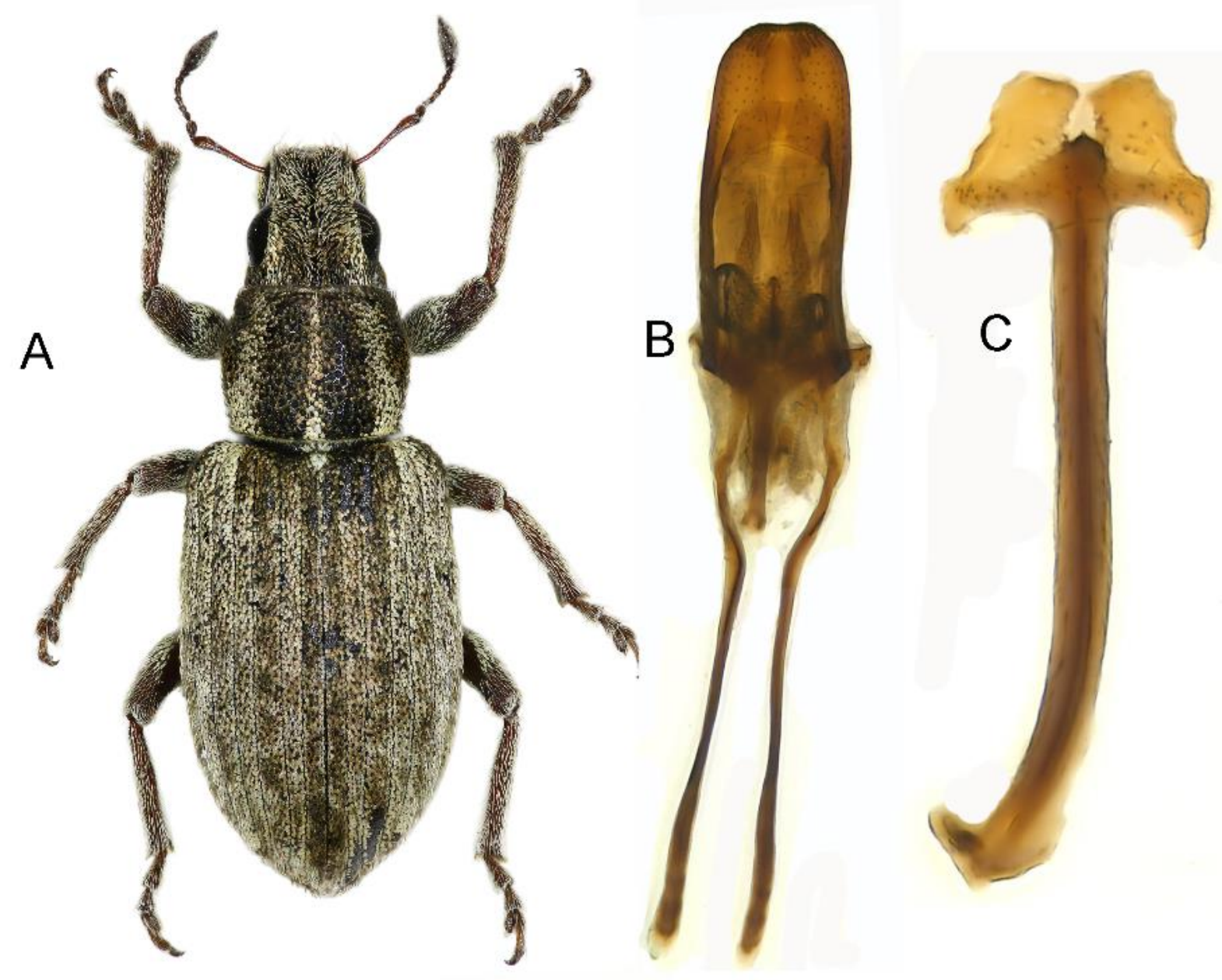

Şekil 1. A- Sitona humeralis Stephens, 1831 (†), B- aedeagus, C- spiculum gastrale

\subsection{Morfoloji}

Dişi ortalama 4,8 mm (4,6 - 5,0 mm), erkek ortalama 4,3 $\mathrm{mm}(3,9-4,5 \mathrm{~mm})$ vücut uzunluğuna sahiptir. Integument siyah, coxa koyu kahverengi, femur, tibia ve anten kahverengidir. Vücudun dorsali çoğunlukla oval (ovate) ve ventrali mızrak (lanceolate) şeklinde parlak açık kahverengi, beyazımsı, sarımsı ve grimsi pul ile yatık kahverengimsi ve beyaz setalarla kaplıdır. Sarımsı, beyazımsı pullar vücudun ventral kısmı, gözün dorsal kenarı, pronotumun dorsalinin ortasında boyuna ince ve lateral kısımlarında ise çok daha geniş bir şerit şeklinde oluşturmaktadır. Bu beyazımsı pullar elytrada scutellum, 5. - 11. interstriaların basal kısmından neredeyse apikale kadar şerit şeklinde uzanmaktadır. Setalar rostrumun dorsal ve apical kısmında, elytranın posterior kısmında yarı kalkık ve daha uzundur. Bu setaların birkaçı kirpik şeklinde göz üzerine uzanmıştır. Hortumun genişliği başın genişliğinden $1 / 2$ katından biraz daha geniş, dorso-lateral kenar belirgin ve kalkık, frons ve epifrons oldukça konkav, median frontal karina mevcut ve fronsun tabanından başın ön kısmına kadar derin bir yarık mevcut, bu yarık interocular pit kısmında oldukça derindir. Bu yarık circum ocular hizasını geçmez. Gözler hafifçe dışarı doğru taşmış, orta kısım hafif konveks yapıda olup, pronotumun apexinden hafifçe dardır. Scape tabanda hafifçe kavisli, uçta genişleyerek topuz oluşturmuş, funicle segmenlerinden 1. segment, 2. segmentin iki katı uzunlukta, oldukça iri ve konik yapıda, 3. - 7. segmentler birbirine benzer hafif konik yapıda, 7. segment biraz daha irice, anten topuzu uca doğru sivrileşen eni boyunun $1 / 3$ kadar ve oblanceolate tiptedir. Her segmentte birkaç seta bulunmaktadır. Prothoraxın eni boyundan biraz dar, apical kısımdan geriye dışa doğru genişlemiş bir şekilde silindirik ve anterior kenarlarına doğru tetricen daralmıştır. Anterior kenarının genişliği elytranın basal kısmının 2/3'ü genişliğindedir. Elytra'nın boyu eninden 1,5x kat daha uzun, lateral kenarları parallel ve geriye doğru daralır, humeri 6. - 9. interstrialar tabanında kaynaşmış ve oldukça belirgindir. Femur tibiaya doğru tedrici şekilde kalınlaşmış pazu şeklinde, uca doğru hafifçe daralır. Tibia hafif kavisli uca doğru tedricen genişlemiş ve uçta tarak şeklinde setalarla mevcuttur. 1. tarsomer 2.'den daha uzun, dorsal ve lateral kısımlarında uca doğru uzanmış uzun setalar bulunmakta (Şekil 1A). Erkek genital organlarından aedeagus, apikal kenara doğru geniş bir şekilde yuvarlanmış ve apical kısmı düzdür (Şekil 1B). Spiculum gastralenin apodeme oldukça kalın ve lamina üstte ayrık, taban kenarları aşağıya doğru uzamıştır (Şekil 1C). 


\subsection{Türkiye'deki yayılışı}

Afyon, Balıkesir, Bilecik, Burdur, Bursa, Çanakkale, Denizli, Isparta, Yalova, İzmir, Kırklareli, Kütahya, Manisa, Muğla, Sakarya, Tekirdağ, Uşak [27]. Adana, Ankara, Antalya, Bartın, Bolu, Çorum, Düzce, Eskişehir, Gaziantep, Hatay, İçel, Kahramanmaraş, Karabük, Karaman, Kayseri, Kırıkkale, Kırşehir, Konya, Nevşehir, Niğde [28]. Ankara [23] Tekirdağ [19], Ankara, Konya [20], Bursa [21].

\subsection{Türkiye'deki yayılışı Dünya'daki yayılışı}

Avrupa: Arnavutluk, Avusturya, Belçika, Bosna Hersek, Bulgaristan, Beyaz Rusya, Hırvatistan, Çek Cumhuriyeti, Danimarka, Estonya, Finlandiya, Fransa (Corse, Monako dahil), Büyük Britanya, Almanya, Yunanistan, Macaristan, İtalya (Sardegna, Sicilia, San Marino dahil), Kazakistan (Ural Nehri'nin batısında), Letonya, Lüksemburg, Makedonya, Moldavya, Hollanda, Norveç, Rusya: Kuzey Avrupa Bölgesi, Polonya, Portekiz, Romanya, Sırbistan, Slovakya, Slovenya, İspanya (Cebelitarık dahil), Rusya: Güney Avrupa Bölgesi, İsveç, İsviçre, Türkiye, Ukrayna, Kuzey Afrika: Madeira Takımadaları (Selvagenler dahil), Asya: Azerbaycan, Ermenistan, Kıbrıs, Gürcistan, İran, Irak, Kırgızistan, Kazakistan (Ural Nehri'nin doğusu), Lübnan, Moğolistan, Mısır: Sina, Suriye, Tacikistan, Türkmenistan, Türkiye, Özbekistan, Rusya: Batı Sibirya [29].

\subsection{Konukçu bitkiler}

Medicago sativa L., Vicia cracca L., V. Faba L., Lens esculenta Medik. [28].

\subsection{Biyolojik notlar}

Sitona humeralis'in ergin, yumurta, larva ve pupası Şekil 2'de gösterilmiştir. Iğdır ili yonca alanlarında $S$. humeralis kışı yumurta, larva ve ergin dönemlerinde geçirdiği belirlenmiştir (03.01.2020 - Çalpala, Halıkışla ve Y. Çarıkçı). Dişi böcekler yumurtalarının bir kısmını sonbaharda ekim-kasım aylarında bırakmakta (17.11.2019Taşburun), hava sıcaklığının düşmesiyle birlikte yumurtlamaya ara vermektedir. Ergin bireyler havaların tekrar 1sınmasıyla birlikte mart sonu-nisan ayının başlarından (27.03.2020 - Yenimahalle, 02.04.2020 - Çalpala) itibaren yonca tarlalarında beslenmeye, çiftleşmeye ve mayıs ayı başlarına kadar yumurta bırakmaya devam etmektedir. Yumurtalarını yonca bitkisinin taç izdüşümünün bulunduğu güneş ışınlarının ulaşamayacağı toprak yüzeyinde toprak ve bitki atıkları arasına koymaktadır. Ekim ayı başlarında bırakılan yumurtalardan çıkan larvalar kasım ayı ortalarına kadar toprak içerisinde aktifken, havaların soğumasıyla şubat ortalarına kadar inaktiftir (03.01.2020 - Çalpala, Halıkışla ve Y. Çarıkçı). Mart başlarında pupa dönemleri tespit edilmiştir (04.03.2020 - Taşburun). Nisan sonu-mayıs başlarında, ilkbaharda bırakılan yumurtalardan çıkan larvalar ise mayıs sonu-haziran ayında pupa dönemine girmektedir. İlkbaharda son dönemde bırakılan yumurtalar haziran - temmuz ayının ortalarında pupa olmakta ve temmuz ortalarında ergin bireyler çıkış yaparak yonca bitkisinin kök bölgesinde ve toprak içerisinde yazı geçirmektedir (18.07.2020 Aşağ1 çiftlik, 21.08.2020 - Çalpala ve 04.09.2020 - Taşburun). Taşburun yonca tarlalarında mayıs sonu yapılan sörveylerde larvalar, yaklaşık $10 \mathrm{~cm}$ derinlikte yonca bitkisinin kök çevresinde bulunmuştur. Larvalar, bulundukları toprak ve bitki kökleriyle birlikte laboratuvara getirilmiştir. Laboratuvarda larvalar ortalama bir ay sonra (09.07.2019) pupa dönemine girmişlerdir. Yonca tarlalarında S. humeralis yumurtalarına mayıs - ekim ayları arasında yapılan toprak örneklemelerinde rastlanılmamıştır. Kışı geçiren erginler nisan - mayıs aylarında doğal ölümler başlamakta, haziran ayından itibaren yeni nesil erginler çıkmaya başlamakta ve bu temmuz ortalarına kadar devam etmektedir. Ağustos’tan eylül sonlarına kadar yeni nesil ergin bireylerin bitki kök boğaz kısmında kitinizasyon ve melanizasyonu tamamlanana kadar bir süre topraktan çıkmadan burada bekledikleri tespit edilmiştir. Mart sonunda (30.03.2020 - Çalpala) laboratuvarda kültüre alınan 5 dişi böcekten toplam mayıs ortalarına kadar (17.05.2020) 1560 yumurta elde edilmiştir (1 dişi bireyden ortalama 312 yumurta). Yumurtaların $0,4 \mathrm{~mm}$ boyunda olduğu ölçülmüştür. Yumurtalar ilk bırakıldığında parlak şeffaf beyaz, daha sonra gri ve siyah renge dönüşmüştür. Ergin bireyler yonca alanlarında en yoğun olarak temmuz ortaları 126.8 birey/10 atrap (16.07.2019) ve ekim ayının 3. haftasında 132 birey/10 atrap (23.10.2019) bulunmuştur.

\section{Sonuçlar ve tartışma}

Çalışmalar sonucunda, Iğdır ili yonca alanlarında $S$. humeralis kışı yumurta, larva ve ergin dönemlerinde geçirdiği belirlenmiştir. Dişi böcekler yumurtalarını, sonbaharda ekim-kasım ve ilkbaharda mart sonu, mayıs aylarında bıraktığı, larvaların ekim - kasım ve nisan - mayıs aylarında aktif olduğu, erginlerin ağustos - ekim ayları arasında yazladı̆̆ı ve yılda bir nesil verdikleri belirlenmiştir. Grigorov [30], Sitona türlerinin Bulgaristan'da yılda bir nesil verdiğini ve kışı farklı biyolojik dönemlerde geçirdiğini bildirmiştir. Pisarek [31], Polonya'da S. humeralis'in yumurtalarının embriyo gelişiminin 10 günde, larva gelişimini 30 günde ve pupa dönemini 14 günde olmak üzere yaşam döngüsünü 54 günde tamamladığını belirtmiştir. Aeschlimann [13], Fransa’nın Tréviers bölgesinde diğer aylara 
kıyasla temmuz - ağustos ayları arası popülasyonun en düşük düzeyde olduğunu raporlamıştır. Papadopoulou [32], Yunanistan'da S. humeralis'in temmuz - ağustos aylarında yazladığı, mart - haziran ve eylül-kasım aylarında çiftleştiği ve yumurta bıraktığı, ekim ayında erginlerin en yüksek sayıya ulaştı̆̆ını ifade etmiştir. Yapılan çalışmaların farklı habitatlarda olmasına rağmen, Iğdır ilinde yürütülen bu çalışma ile benzerlik göstermektedir. S. humeralis yonca alanlarında öne çıkan bir tür olması, onun birçok iklim koşullarına uyum sağlama yeteneğinin olduğunu göstermektedir [33]. Bununla birlikte, yonca bitkisinde, larvalarının bitki kökünde beslenmesi ve ergin vücutlarının küçük olması nedeniyle yonca yetiştiricilerinin gözünden kaçmaktadır. Bu yüzden $S$. humeralis üzerinde daha detaylı biyolojik ve zararıyla ilgili araştırmaların yürütülmesi gerekmektedir.

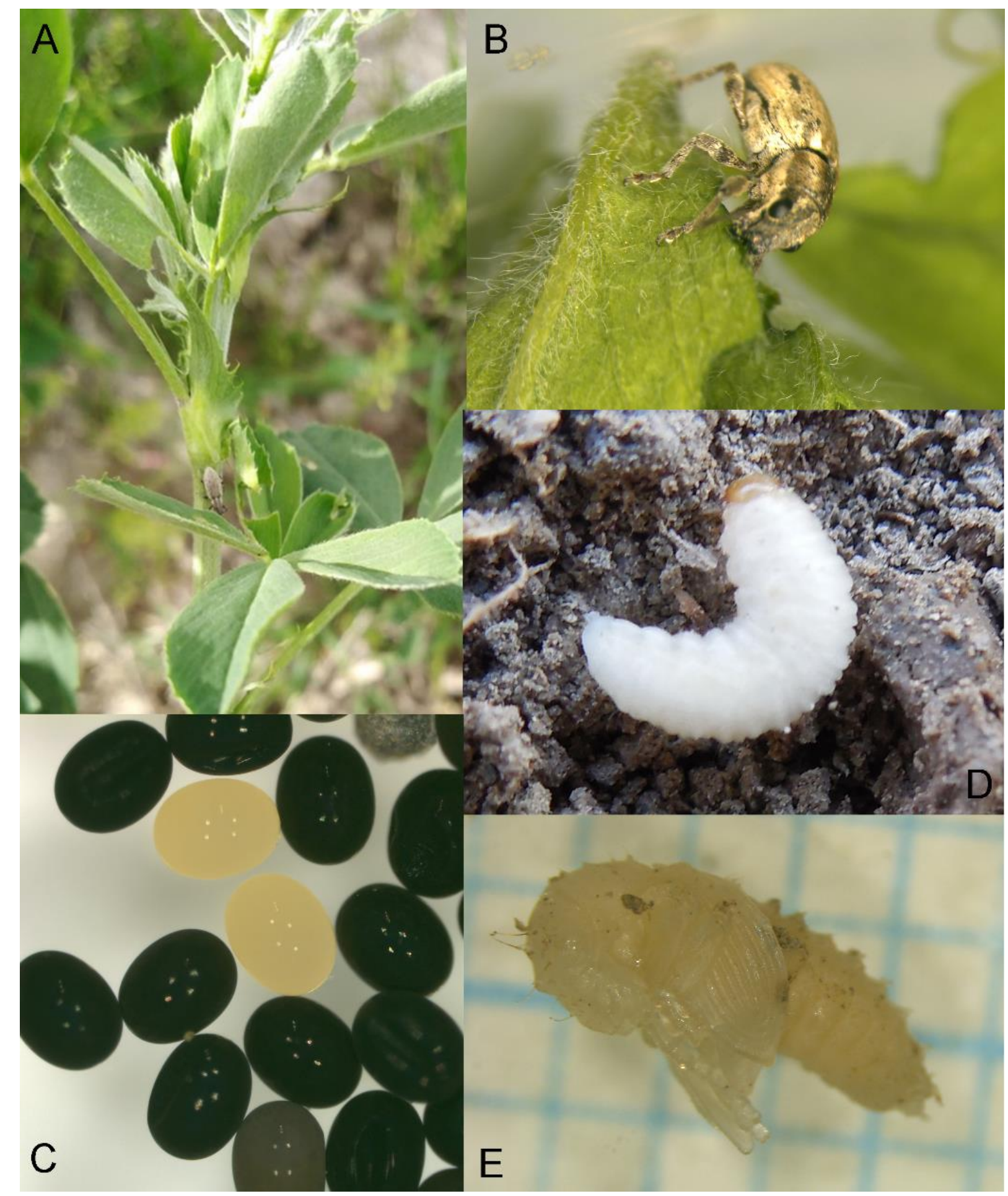

Şekil 2. A, B- Sitona humeralis Stephens, 1831 (ergin), C- yumurta, D- larva, E-pupa 


\section{Kaynaklar}

[1] Davis, P. H. (1978). Flora of Turkey and the east Aegean islands (vol. 6). Edinburgh, SCT: Edinburgh University Press.

[2] Radovic, J., Sokolovic, D., \& Markovic, J. (2009). Alfalfa-most important perennial forage legume in animal husbandry. Biotechnology in Animal Husbandry, 25(5-6), 465-475. doi.org/10.2298/BAH0906465R

[3] Bitkisel üretim istatistikleri. (2019). Ankara, TR: https://biruni.tuik.gov.tr/medas/?kn=92\&locale=tr.

[4] Jackson, D. J. (1921). Bionomics of the weevils of the genus Sitones injurious to leguminous crops in Britain. Part I. Annals of Applied Biology, 7, 269-298. doi.org/10.1111/j.1744-7348.1920.tb05310.x

[5] Danthanarayana, W. (1967). Host specificity of Sitona beetles. Nature, 213, 1153-1154.

[6] Plaut, H. N. (1976). On the biology and phenology of the preimaginal stages of Sitona limosus Rossi (Col. Curculionidae) in Israel. Zeitschrift für Angewandte Entomologie, 78, 381-386.

[7] El-Dessouki, S., \& El-Awady, S. (1978). Studies on the development and bionomy of Sitona lividipes Fhs. (Col., Curculionidae). Zeitschrift für Angewandte Entomologie, 85, 275-280. doi.org/10.1111/j.14390418.1978.tb04039.x

[8] Aeeschlimann, J. P. (1984). Distribution, host plants and reproductive biology of the Sitona humeralis Stephens group of species (Coleoptera, Curculionidae). Zeitschrift für Angewandte Entomologie, 98 (3), 298-309.

[9] Syrett, P. (1992). Feeding range of Sitona regensteinensis Hbst. (Coleoptera: Curculionidae), a potential agent for biological control of Cytisus scoparius (L.) Link (broom) in New Zealand. Biocontrol Science and Technology, 2, 261-265. doi.org/10.1080/09583159209355240

[10] Skot, L., Timms E., \& Mytton, L. R. (1994). The effect of toxin-producing Rhizobium strains, on larvae of Sitona flavescens feeding on legume roots and nodules. Plant and Soil, 163(1), 141-150.

[11] Murray, P. J., \& Clement R. O. (1998). Investigations of the host feeding preferences of Sitona weevils found commonly on white clover (Trifolium repens) in the UK. Entomologia Experimentalis et Applicata, 71(1), 7379. Doi: $\underline{10.1007 / \mathrm{BF} 02380571}$

[12] Cantot, P. (2001). Influence de quelques Papilionacées sur la ponte et le développement larvaire de Sitona lineatus (L.) (Coleoptera, Curculionidae). Bulletin de la Societe Entomologique de France, 1065, 441-447.

[13] Aeschlimann, J. P. (1980). The Sitona (Coleoptera, Curculionidae) species occurring on Medicago and their natural enemies in the Mediterranean region. Entomophaga. 25, 139-153.

[14] Goldson, S. L., Frampton, E. R., Barratt, B. I. P., \& Ferguson, C. M. (1984). The seasonal biology of Sitona discoideus Gyllenhal (Coleoptera: Curculionidae), an introduced pest of New Zealand lucerne. Bulletin of Entomological Research, 74, 249-259.

[15] Goldson, S. L., Bourdot, G. W., \& Proffitt, J. R. (1987). A study of the effects of Sitona discoideus (Coleoptera: Curculionidae) larval feeding on the growth and development of lucerne (Medicago sativa). Journal of Applied Ecology, 24, 153-161.

[16] Dimitrov, D., Kontev, H., \& Lecheva, I. (2006). Investigations on harmful and beneficial entomofauna in field peas under the conditions of Dobroudja region. Plant science, 43(5), 387-391.

[17] Dimitrov, D., Kontev, H., \& Lecheva, I. (2008). Investigations on harmful and beneficial entomofauna in common spring vetch under the conditions of Dobroudja region. Plant science, 45(1), 11-15.

[18] Özbek, H. (1986). Erzurum'da yoncadaki böcek faunasının tespiti. Atatürk Üniversitesi Ziraat Fakültesi Ziraat Dergisi, 17(1-4), 1-20.

[19] Kivan, M., 1995. Tekirdağ ilinde baklagil yem bitkilerinde bulunan Sitona Gm. (Coleoptera, Curculionidae) türleri, konukçuları ve yayılışları üzerine ön araştırmalar. Türkiye Entomoloji Dergisi, 19(4), 299-304.

[20] Tamer, A., Has, A., Aydemir, M., \& Çalışkaner, S. (1997). Orta Anadolu Bölgesinde yemeklik baklagiller (mercimek, nohut, fasulye)'de görülen zararlı ve faydalı böcekler. Bitki Koruma Bülteni, 38 (1-2), 65-90.

[21] Coşkuncu, K., \& Gencer, N. (2010). Determination of the species of Curculionoidea superfamily on alfalfa fields (Medicago sativa L.) and their distribution in Bursa province of Turkey. Journal of Biological \& Environmental Sciences, 4(12): 123-131.

[22] Kaya, K. (2018). Hatay ili yonca üretim alanlarında bulunan böcek faunasının tespiti ve bazı türlerin popülasyon yoğunluklar1. Türk Tarım - Gida Bilim ve Teknoloji Dergisi, 6(3): 352-359. Doi: 10.24925/turjaf.v6i3.352359.1747

[23] Sert, O., \& Çağatay, N. (1994). Systematic studies on the some species of the genera Sitona, Bangasternus and Larinus (Coleoptera: Curculionidae). Turkish Journal of Entomology, 18(4), 223- 236.

[24] Aeschlimann, J. P. (1975). A method for the extraction of Sitona (Col: Curculionidae) eggs from soil and occurrence of a Mymarid (Hym: Chalcidoidea) in the Mediterranean region. Entomophaga, 20(4), 405-408.

[25] Aeschlimann, J. P. (1979). Sampling methods and construction of life tables for Sitona humeralis populations (Col., Curculionidae) in Mediterranean climatic areas. Journal of Applied Ecology, 16, 405-415.

[26] Velázquez de Castro, A., Alonso-Zarazaga, M. A., \& Outerelo, R. (2007). Systematics of Sitonini (Coleoptera: Curculionidae: Entiminae), with a hypothesis on the evolution of feeding habits. Systematic Entomology, 32 , 312-331. 
[27] Velázquez de Castro, A., Friedman, A. L. L., \& Borovec, R., 2010. Sitonini of Israel (Coleoptera: Curculionidae: Entiminae). Israel Journal of Entomology, 40: 71-108.

[28] Lodos, N., Önder, F., Pehlivan, E., \& Atalay, R., 2003. Faunistic studies on Curculionidae (Coleoptera) of Western Black Sea, Central Anatolia and Mediterranean Regions of Turkey. İzmir: Meta Basım.

[29] Alonso-Zarazaga, M. A., Barrios, H., Borovec, R., Bouchard, P., Caldara, R., Colonnelli, E...Yunakov, N. N. (2017). Cooperative Catalogue of Palaearctic Coleoptera Curculionoidea. Retrieved from http://weevil.info/content/palaearctic-catalogue.

[30] Grigorov, S. P. (1972). Special entomology. Sofia, BU: Zemisdat.

[31] Pisarek, M. (2001). The effects of Sitona humeralis larvae on alfalfa Medicago sativa (L.) development. Journal of Plant Protection Research, 41: 52-56.

[32] Papadopoulou, S. (2013). Determination of insecticide application time in alfalfa crops against Sitona humeralis Stephens, based on its biology and ethology. Biotechnology \& Biotechnological Equipment, 27(2), 3665-3668. Doi: 10.5504 / BBEQ.2013.0014

[33] Özdemir, S., Özkan, K. \& Mert, A. (2020). An ecological perspective on climate change scenarios. Biological Diversity and Conservation. 13(3), 361-371. Doi: 10.46309/Biodicon.2020. 762985 